\title{
The GPR40 Full Agonist SCO-267 Improves Liver Parameters in a Mouse Model of Nonalcoholic Fatty Liver Disease without Affecting Glucose or Body Weight
}

\author{
Mitsugi Ookawara, Keisuke Matsuda, Masanori Watanabe, and (1) Yusuke Moritoh \\ SCOHIA PHARMA, Inc., Kanagawa, Japan
}

Received April 11, 2020; accepted July 21, 2020

\begin{abstract}
Full agonism of G-protein-coupled receptor 40 (GPR40)/free fatty acid 1 receptor improves glycemic control in diabetic rodents. However, the effects of GPR40 full agonism on liver parameters are largely unknown. In the present study, we examined the effects of a GPR40 full agonist, SCO-267, on liver parameters in a nondiabetic mouse model with early-stage nonalcoholic fatty liver disease (NAFLD). SCO-267 was orally administered to mice, which were fed a choline-deficient, L-amino acid-defined, high-fat diet (CDAHFD), a mouse model for NAFLD. An oral dose of SCO-267 increased levels of circulating glucagon and glucagon-like peptide1 in CDAHFD-fed mice. In a chronic-dose experiment, effects of SCO-267 were compared with those of a dipeptidyl peptidase-4 inhibitor (alogliptin) and a sodium glucose cotransporter 2 inhibitor (dapagliflozin). SCO-267 decreased liver triglyceride content, weight, collagen content, and plasma alanine aminotransferase (ALT) levels without affecting food intake or glucose levels in CDAHFD-fed mice. Furthermore, SCO-267
\end{abstract}

decreased levels of liver thiobarbituric acid reactive substances (TBARS), markers of oxidative stress. Alogliptin and dapagliflozin had no effect on liver weight or levels of triglyceride, collagen, plasma ALT, and liver TBARS. SCO-267 elevated mRNA levels of molecules with roles in mitochondrial function and $\beta$-oxidation while inhibiting those with roles in lipogenesis, inflammation, reactive oxygen species generation, and fibrosis in the liver, all of which were less evident with alogliptin and dapagliflozin. This is the first study to show that the GPR40 full agonist SCO-267 improves liver parameters without affecting glucose or body weight in a mouse model of NAFLD.

\section{SIGNIFICANCE STATEMENT}

Full agonism of GPR40/free fatty acid 1 receptor signaling stimulates islet and gut hormone secretions. The present study is the first to show the treatment effects of GPR40 full agonism on liver parameters in a mouse model for nonalcoholic fatty liver disease.

\section{Introduction}

Nonalcoholic fatty liver disease (NAFLD) is a spectrum of chronic liver diseases ranging from noninflammatory isolated steatosis, characterized by triglyceride accumulation in hepatocytes, to nonalcoholic steatohepatitis (NASH), a more advanced form of the disease, which is characterized by steatosis, inflammation, and hepatocyte cell ballooning associated with liver fibrosis (Arab et al., 2018). NAFLD is a major cause of other liver disease worldwide and will likely emerge as the leading cause of end-stage liver disease in the future. To date, weight loss and lifestyle changes are considered to be effective ways to overcome NAFLD (Wattacheril et al., 2018). In addition, because of the lack of approved drugs, there is an urgent need for development of effective pharmacological treatments for patients with NAFLD (Wattacheril et al., 2018). The pathogenesis of NAFLD is complex and still unclear. Although understanding of the molecular

This work was supported by SCOHIA PHARMA, Inc https://doi.org/10.1124/jpet.120.000046.

S This article has supplemental material available at jpet.aspetjournals.org. mechanisms regulating disease development and progression has grown significantly over recent years, the detailed contribution of genetic and environmental factors, as well as that of intrahepatic and extrahepatic events, in determining the disease conditions remains poorly defined (Musso et al., 2016). These factors hinder the development of new drugs for the treatment of NAFLD.

G-protein-coupled receptor 40 (GPR40)/free fatty acid 1 receptor (FFAR1) primarily couples with the Gq/11 protein, facilitating phospholipase C-mediated hydrolysis of phosphatidylinositol 4,5-bisphosphate into diacylglycerol and inositol 1,4,5-triphosphate (Ghislain and Poitout, 2017). GPR40 is expressed in pancreatic islet and intestinal endocrine cells and regulates hormone secretions from both sites (Mancini and Poitout, 2013; Pais et al., 2016). The recent discovery of synthetic GPR40 full agonists confirmed the significant role of GPR40 activation in stimulating glucagon, an islet hormone, and glucagon-like peptide-1 (GLP-1), a gut hormone (Ueno et al., 2019). GPR40 activation is confirmed to be effective in alleviating diabetes in rodents and humans (Kaku et al., 2016; Ueno et al., 2019). Recently, clinical trials investigating GLP-1 receptor agonists for the treatment of NAFLD have shown

ABBREVIATIONS: ALT, alanine transferase; CDAHFD, choline-deficient; L-amino acid-defined, high-fat diet; GLP-1, glucagon-like peptide-1; GPR40, G-protein-coupled receptor 40; NAFLD, nonalcoholic fatty liver disease; NASH, nonalcoholic steatohepatitis; TBARS, thiobarbituric acid reactive substances. 
therapeutic potential (Armstrong et al., 2016). In addition, injectable GLP-1 receptor/glucagon receptor dual agonists are being investigated for the treatment of NAFLD (Patel et al., 2018), indicating the therapeutic potential of combining islet and gut hormones to treat NAFLD. The ability of GPR40 full agonists to increase levels of glucagon and GLP-1 warrants further investigation into the effects of this class of compounds on NAFLD.

This study is the first to report the therapeutic potential of a GPR40 full agonist, SCO-267 (Ueno et al., 2019), on liver parameters in nondiabetic mouse models of early-stage NAFLD. In the current study, we used a choline-deficient, L-amino acid-defined, high-fat diet (CDAHFD)-induced mouse model of NAFLD (Matsumoto et al., 2013). Initially, the glucagon- and GLP-1-elevating effects of SCO-267, a GPR40 full agonist, were evaluated in CDAHFD-fed mice. Subsequently, the effects of chronic dosing of SCO-267 were evaluated in CDAHFD-fed mice. In addition, certain key effects induced by this GPR40 full agonist were compared with those of a dipeptidyl peptidase- 4 inhibitor (alogliptin) (Keating, 2015) and a sodium glucose cotransporter 2 inhibitor (dapagliflozin) (Filippatos et al., 2015). Both of these drugs represent different classes of orally available antidiabetic drugs used for the treatment of metabolic dysfunction, a risk factor for NAFLD (Kothari et al., 2019), and have been reported to have glucose-independent therapeutic effects (Uchii et al., 2016; McMurray et al., 2019). However, the roles of these drugs in reversing nondiabetic NAFLD are poorly understood.

\section{Materials and Methods}

Materials. All reagents were purchased from Sigma-Aldrich (Tokyo, Japan), FUJIFILM Wako Pure Chemical Corporation (Osaka, Japan), or Cayman Chemical (Ann Arbor, MI) unless otherwise indicated. SCO-267, alogliptin, and dapagliflozin were obtained from SCOHIA PHARMA, Inc. For in vivo studies, compounds were suspended in $0.5 \%(\mathrm{w} / \mathrm{v})$ methylcellulose solution (FUJIFILM Wako).

Animals. All animals were housed in a room with controlled temperature $\left(23^{\circ} \mathrm{C}\right)$, humidity $(55 \%)$, and lighting (12-hour light/dark cycle). All animals were provided ad libitum access to a standard laboratory chow diet (CE-2, $3.4 \mathrm{kcal} / \mathrm{g}, 12 \mathrm{kcal} \%$ fat, $59 \mathrm{kcal} \%$ carbohydrate, and $30 \mathrm{kcal} \%$ protein; CLEA Japan, Inc., Tokyo, Japan) and tap water during the acclimation period. The care of the animals and use of the experimental protocols in the current studies were approved by the Institutional Animal Care and Use Committee in Shonan Health Innovation Park, accredited by the American Association for Accreditation of Laboratory Animal Care. We used 0.5\% methylcellulose as the vehicle in animal studies. Blood samples were obtained from the tail vein of the animals. Blood samples were centrifuged $\left(13,000 \mathrm{~g}, 4^{\circ} \mathrm{C}, 5\right.$ minutes $)$, and plasma samples were used for subsequent analyses.

Single-Dose Study of SCO-267 in CDAHFD-Fed Mice. Male C57/BL6J mice, obtained from CLEA Japan, Inc., were fed a CDAHFD (Matsumoto et al., 2013) (5.2 kcal/g, $62 \mathrm{kcal} \%$ fat, $20 \mathrm{kcal} \%$ carbohydrate, and $18 \mathrm{kcal} \%$ protein, and $0.1 \%$ methionine, A06071302; Research Diets, Inc.) for 1 week prior to beginning the study. For the determination of glucagon and GLP-1 levels in the nonfasted state, 12 week-old CDAHFD-fed mice were randomized into groups $(n=6)$ based on their body weight. The test compounds were orally administered at the indicated dose levels. Blood was collected at the indicated time points, and plasma parameters were determined. A pharmacokinetic study was similarly performed using nonfasted, 15-week-old, CDAHFD-fed mice $(n=4)$.
Repeated-Dose Study of SCO-267 in CDAHFD-Fed Mice. Male C57/BL6J mice, obtained from CLEA Japan, Inc., were fed CDAHFD for 1 week before the start of the study. For the chronicdosing study, 11-week-old CDAHFD-fed mice (baseline body weight, $25.4 \pm 1.6 \mathrm{~g}$ ) were randomized into groups $(n=8)$ based on their body weight, plasma alanine aminotransferase (ALT), aspartate aminotransferase, and glucose levels. Mice were then orally dosed with SCO$267(3,10 \mathrm{mg} / \mathrm{kg}$, twice a day), alogliptin $(10 \mathrm{mg} / \mathrm{kg}$, twice a day), dapagliflozin $(1 \mathrm{mg} / \mathrm{kg}$, twice a day), or vehicle alone at approximately 8-10 AM for the first dose and 4-6 PM for the second dose for 4 weeks. Food intake and body weight were measured regularly. Male C57/ BL6J mice (baseline body weight, $25.3 \pm 0.8 \mathrm{~g}$ ) in the normal control group were fed a standard diet (CE-2; CLEA Japan, Inc.) and received vehicle during the experimental period. Plasma glucose, triglyceride, alanine aminotransferase, and insulin levels were determined after 4 weeks of drug dosing. At the end of the study, the mice were euthanized with isoflurane (3\%), and their livers were isolated, weighed, divided, and immediately frozen for measurements of liver triglyceride, thiobarbituric acid reactive substance (TBARS), and mRNA levels.

Plasma Measurements. Plasma parameters were determined using Autoanalyzer 7180 (Hitachi, Tokyo, Japan). Enzyme-linked immunosorbent assay kits were used to measure total plasma levels of insulin (MS301; Takara, Shiga, Japan), glucagon (10-1271-01; Mercodia, Uppsala, Sweden), and total GLP-1 (299-75501; FUJIFILM Wako). The amount of total collagen in the liver was quantified using a QuickZyme Total Collagen Assay kit (QuickZyme Biosciences, Leiden, The Netherlands).

Measurement of Hepatic Triglyceride Content. Liver tissue was weighed and placed in a polypropylene tube. A 5-fold (v/w) volume of saline was added to the tube, and samples were homogenized on ice. The homogenate $(0.5 \mathrm{ml})$ was mixed with $1 \mathrm{ml}$ of extraction solvent (hexane: isopropanol $=3: 2, \mathrm{v} / \mathrm{v}$ ) and shaken for 10 minutes. The samples were centrifuged ( $800 \mathrm{~g}, 5$ minutes, room temperature), and the supernatant was transferred to clean tubes. The bottom layer was again mixed with extraction solvent $(0.5 \mathrm{ml})$, and the supernatant was prepared in a similar manner. The supernatant layers containing the extracted lipid were then mixed and dried under a stream of nitrogen gas. The dried sample was dissolved in isopropanol. The concentrations of triglyceride were measured using a Triglyceride E-test (FUJIFILM Wako).

Measurement of TBARS Content. Lipid peroxidation is a well established mechanism of cellular injury in animals and is used as an indicator of oxidative stress in cells and tissues. The measurement of TBARS content is a well established method for monitoring lipid peroxidation (Yagi, 1998). For the measurement of tissue TBARS levels, radioimmunoprecipitation assay buffer containing cOmplete Mini Ethylene Diaminetetraacetic Acid-Free Protease Inhibitor Cocktail (4693159; Roche) was added to liver samples. The samples were then homogenized and centrifuged $\left(1600 \mathrm{~g}, 4^{\circ} \mathrm{C}, 10\right.$ minutes). TBARS levels of the samples were determined using a TBARS (TCA Method) Assay Kit (700870; Cayman Chemical).

Measurement of mRNA Levels. Liver RNA samples were prepared using QIAzol Lysis Reagent (Qiagen, Venlo, Netherlands) and RNeasy Mini Kit (Qiagen). First-strand cDNA was synthesized by using ReverTra Ace qPCR RT Master Mix (Toyobo, Osaka, Japan). Quantitative polymerase chain reaction was performed using the TaqMan gene expression assay (Supplemental Table 1, Uniprot entry included) and THUNDERBIRD Probe qPCR Mix (Toyobo) or primers (Supplemental Table 2, Uniprot entry included) and THUNDERBIRD SYBR qPCR Mix on the ABI PRISM 7900HT Sequence detector. Expression relative to the vehicle group was calculated by using the comparative CT $(\Delta \Delta \mathrm{CT})$ method (User Bulletin \#2; Applied Biosystems). For the comparative CT method, the $\Delta \mathrm{CT}$ value was determined by subtracting the average of internal control (glyceraldehyde-3phosphate dehydrogenase, Gapdh) CT value from the average of target CT value.

Statistical Analysis. Statistical significance was first analyzed using Bartlett's test for homogeneity of variances, followed by the 
Williams' test $(P>0.05)$ and Shirley-Williams test $(P \leq 0.05)$ for dosedependent studies. Alternatively, statistical significance was analyzed using the $\mathrm{F}$ test for homogeneity of variances, followed by Student's $t$ test $(P>0.2)$ or the Aspin-Welch test $(P \leq 0.2)$. The Williams' and Shirley-Williams tests were conducted using a onetailed significance level of $2.5 \%$ (0.025). Other tests were conducted using a two-tailed significance level of $1 \%(0.01)$ and $5 \%$ (0.05). All data are presented as means \pm S.D.

\section{Results}

SCO-267 Stimulated Glucagon and GLP-1 Secretion in CDAHFD-Fed Mice. To determine the effects of SCO-267 on glucagon and GLP-1 secretion in CDAHFD-fed mice, the compound was orally administered, and changes in these hormone levels were measured. Consistent with a previous report in rats (Ueno et al., 2019), SCO-267 was also found to significantly $(P<0.025)$ stimulate glucagon and GLP-1 secretion in CDAHFD-fed mice (Fig. 1). Based on the effective elevation of glucagon and GLP-1 by a single dose of SCO-267, we performed the subsequent chronic-dose study in CDAHFD-fed mice.

SCO-267 Did Not Alter Food Intake, Body Weight, and Glucose Control in CDAHFD-Fed Mice. Because of the relatively short half-life of each compound in mice (Supplemental Fig. 1), and based on previous reports (Zhang et al., 2011; Tahara et al., 2016), we dosed SCO-267 (3 and
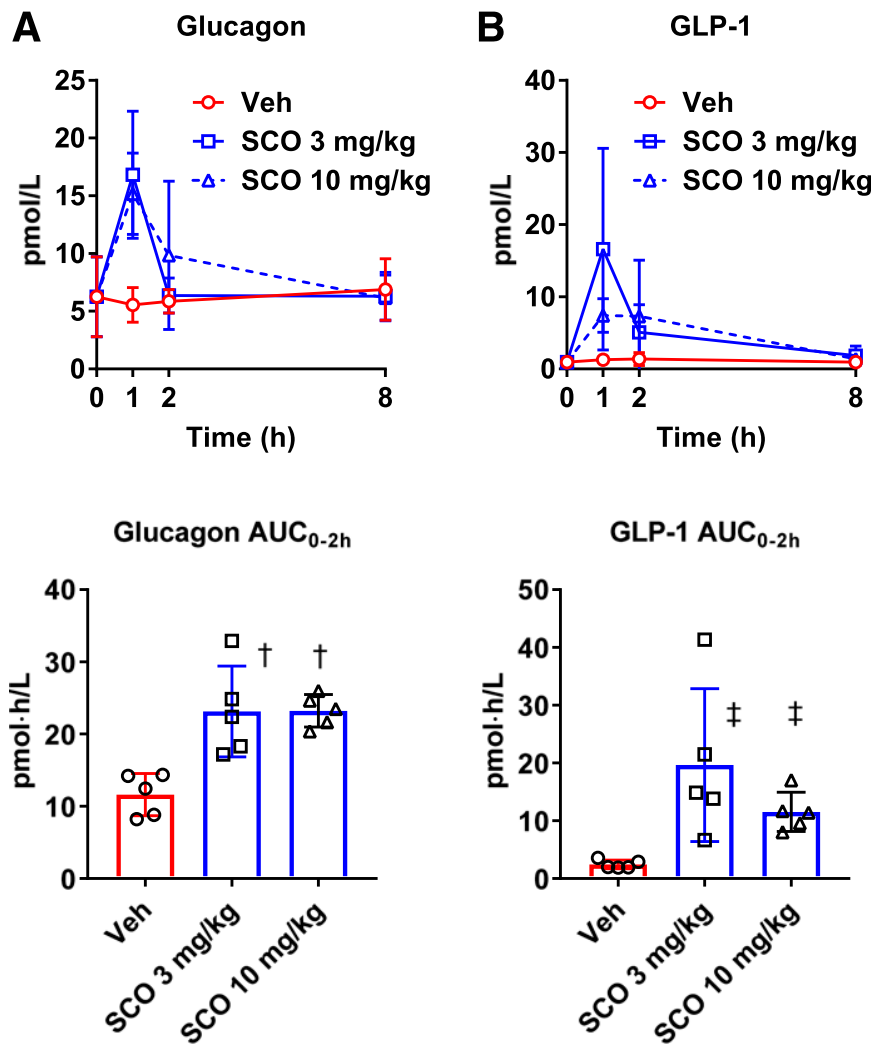

Fig. 1. Effects of SCO-267 on glucagon and GLP-1 secretion in CDAHFDfed mice. (A) Glucagon levels and (B) GLP-1 levels after drug dosing. SCO267-stimulated glucagon and GLP-1 secretion in CDAHFD-fed mice. Values are presented as means \pm S.D. $(n=5) .{ }^{\dagger} P<0.025$ and ${ }^{\ddagger} P<0.025$ vs. vehicle by one-tailed Williams' test and one-tailed Shirley-Williams test, respectively. $\mathrm{AUC}_{0-2}$ h, area under the curve from 0 to 2 hours; SCO, SCO-267; Veh, vehicle.
$10 \mathrm{mg} / \mathrm{kg})$, alogliptin $(10 \mathrm{mg} / \mathrm{kg})$, and dapagliflozin $(1 \mathrm{mg} / \mathrm{kg})$ twice a day in a repeated-dose study in CDAHFD-fed mice. As shown in Fig. 2A, SCO-267 and alogliptin did not affect food intake, whereas dapagliflozin slightly increased this parameter. Body weight changes were within a similar range for SCO-267, alogliptin, and dapagliflozin (Fig. 2B). The plasma glucose level was unchanged in SCO-267- and dapagliflozintreated mice, whereas alogliptin slightly elevated this parameter (Fig. 2C). SCO-267, alogliptin, and dapagliflozin had no effect on plasma triglyceride levels (Fig. 2D). Plasma insulin levels were unaltered by the treatment with SCO-267, alogliptin, and dapagliflozin (Supplemental Fig. 2).

SCO-267 Decreased Liver Triglyceride Content, Liver Weight, Liver Collagen Levels, and Plasma ALT Levels in CDAHFD-Fed Mice. Liver-related parameters were determined at the end of the 4-week study. Of note, SCO267 significantly $(P<0.025)$ decreased liver triglyceride content and weight in a dose-dependent manner (Fig. 3, A and B). Furthermore, SCO-267 significantly $(P<0.025)$ decreased liver collagen content and plasma ALT levels in CDAHFD-fed mice in a dose-dependent manner (Fig. 3, C and D). Alogliptin and dapagliflozin did not induce similar effects in this model (Fig. 3). Plasma aspartate aminotransferase levels were unchanged in SCO-267-, alogliptin-, and dapagliflozintreated mice (Supplemental Fig. 3).

SCO-267 Decreased Oxidative Stress Marker TBARS Levels in the Liver. Excessive oxidative stress in the liver is considered an aggravating factor for NAFLD (Spahis et al., 2017). As SCO-267 significantly $(P<0.025)$ decreased liver triglyceride content, we further quantified the oxidative stress marker TBARS in the liver of this mouse model. Interestingly, SCO-267 potently decreased liver TBARS levels, whereas alogliptin and dapagliflozin did not cause any significant change in liver TBARS levels (Fig. 4).

SCO-267 Restored Dysregulated mRNA Expression in Liver. Liver samples isolated at the end of the study were further evaluated with respect to the levels of mRNAs regulating liver functions. SCO-267 increased mRNA levels of transcription factor A, mitochondrial (Tfam), a transcription factor regulating mitochondrial function (Fig. 5A), as well as peroxisome proliferator-activated receptor $\alpha$ (Ppara) and acyl-CoA dehydrogenase, long chain (Acadl), both gene products involved in the $\beta$-oxidation pathway (Fig. 5B). In contrast, SCO-267 decreased mRNA levels of sterol regulatory element binding transcription factor 1 (Srebf1) and CD36 molecule (Cd36) of the lipogenic pathway (Fig. 5C), as well as tumor necrosis factor (Tnf), chemokine (C-C motif) ligand 2 (Ccl2), interleukin-6 (Il6), and transforming growth factor $\beta 1$ (Tgfb1) of the inflammatory pathway (Fig. 5D). Furthermore, SCO267 downregulated the expression of neutrophil cytosol factor 1 (Ncf1), and cytochrome b-245 and $\beta$ polypeptide ( $C y b b)$ (Fig. 5E), both of which contribute to the generation of reactive oxygen species, and collagen type $1 \alpha 1$ (Col1a1) and actin, $\alpha 2$, smooth muscle, aorta (Acta2) (Fig. 5F), which are both fibrosiscausing factors. SCO-267 treatment also significantly $(P<$ 0.025) decreased mRNA levels of glucose-6-phosphatase, catalytic subunit $(G 6 p c)$; however, it increased the mRNA levels of glycogen synthase 2 (Gys2), both molecules of the glucose metabolism pathway (Supplemental Fig. 4). Most of the beneficial changes observed were more prominent in the SCO-267 group than in the alogliptin and dapagliflozin groups (Fig. 5). Lastly, we assessed mRNA expression for GPR40 
A
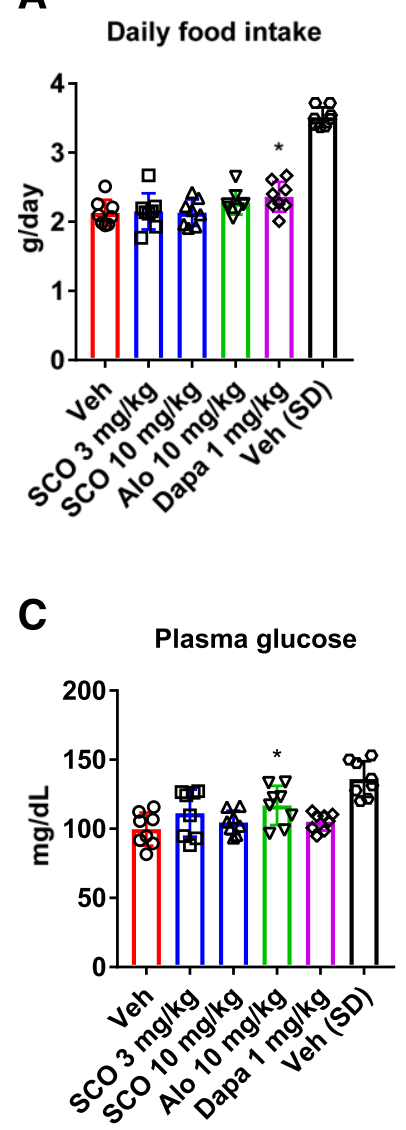

B

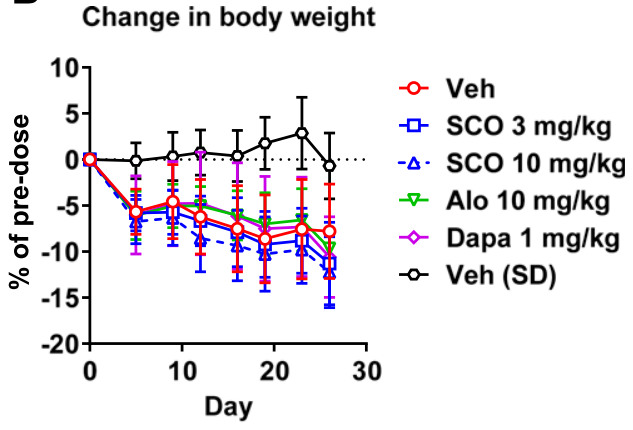

D

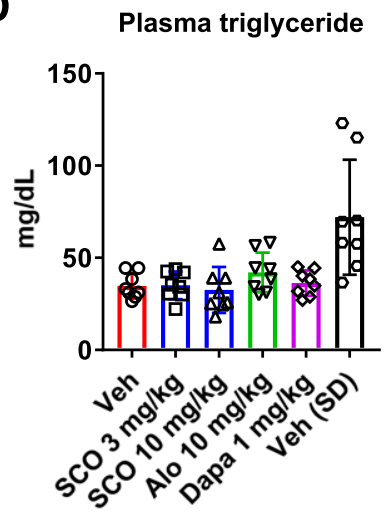

Fig. 2. Effects of repeated doses of SCO-267 on metabolic parameters in CDAHFD-fed mice. (A) Daily food intake, (B) change in body weight during the study, (C) plasma glucose, and (D) plasma triglyceride at the end of the 4-week study. Baseline body weight for CDAHFD group was $25.4 \pm 1.6 \mathrm{~g}$. SCO-267 did not affect food intake, body weight, plasma glucose, or plasma triglyceride levels. Values are presented as means \pm S.D. $(n=8)$. ${ }^{*} P<0.05$ vs. vehicle by Student's $t$ test. Alo, alogliptin benzoate; Dapa, dapagliflozin; SCO, SCO-267; SD, standard diet; Veh, vehicle.
(Ffar 1 ) and confirmed that Ffar 1 was not expressed in livers obtained from CDAHFD-fed and standard diet-fed mice (Supplemental Fig. 5; Supplemental Table 3).

\section{Discussion}

GPR40 is a key receptor regulating pancreatic and gut hormone secretion. In this study, effects of SCO-267, a GPR40 full agonist, on liver-related parameters were evaluated in CDAHFD-fed mice, a nondiabetic animal model for NAFLD. To the best of our knowledge, this report is the first to demonstrate that a GPR40 full agonist improved abnormal liver-related conditions in a preclinical NAFLD disease model. Of note, SCO-267 improved liver-related parameters without affecting glucose levels or body weight. This suggests that improvement in glycemic parameters and body weight control need not be a primary driver for the improvement of liverrelated parameters in SCO-267-treated CDAHFD-fed mice. Hence, GPR40 full agonism may be used for the treatment of NAFLD with or without diabetes and obesity.

The effective secretion of glucagon after SCO-267 treatment suggests that this hormone may have contributed to the treatment effect of SCO-267 in the present study. Glucagon is highly expressed in the liver and shows diverse physiologic effects in the body (Svoboda et al., 1994). Besides its pivotal role in liver glucose metabolism, glucagon controls liver fat metabolism by stimulating lipid oxidation and decreasing lipid synthesis (Seghieri et al., 2018). In fact, SCO-267 treatment decreased liver mRNA levels of Srebf1 and $C d 36$ of the lipogenesis pathway. However, it increased that of Tfam involved in mitochondria function, and of Ppara and $A c a d l$ involved in $\beta$-oxidation. Furthermore, a recent study demonstrated that glucagon receptor signaling is necessary for the regulation of hepatocyte survival, as it exerts antiapoptotic effects in the liver (Sinclair et al., 2008). Thus, SCO-267-induced activation of liver glucagon signaling may directly improve hepatic disease conditions in this NAFLD model.

In addition to the decreased liver fat, SCO-267 decreased inflammation and fibrosis markers in CDAHFD-fed mice. Based on the "multiple parallel hit" hypothesis, NAFLD pathogenesis is considered a sequence of events from simple steatosis to hepatic inflammation toward fibrosis and NASHassociated hepatocellular carcinoma (Cohen et al., 2011). Thus, SCO-267-induced decrease of hepatic steatosis may be the underlying cause of the reduction in hepatic inflammation/ fibrosis by SCO-267 in the present study.

Oxidative stress has been recognized as a central mechanism contributing to liver lesions, as it accelerates the transition from simple steatosis to NASH (Spahis et al., 2017). In fact, the Pioglitazone versus Vitamin E versus Placebo for the Treatment of Nondiabetic Patients with Nonalcoholic Steatohepatitis (PIVENS) trial studying pioglitazone versus vitamin $\mathrm{E}$ versus placebo in adults with NASH and without diabetes demonstrated that vitamin E, a lipid-soluble antioxidant, led to improvement in liver histologic features and significant resolution of NASH (Sanyal et al., 2010). This demonstrated that reducing 
A
Liver triglyceride content

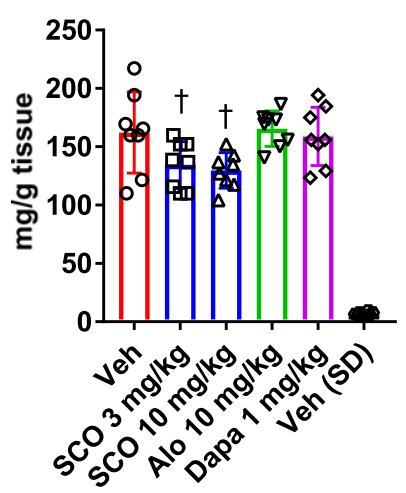

C

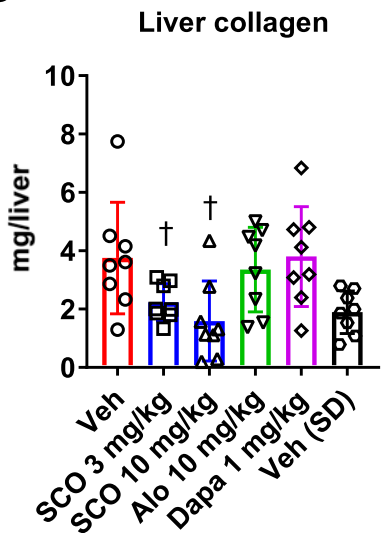

B

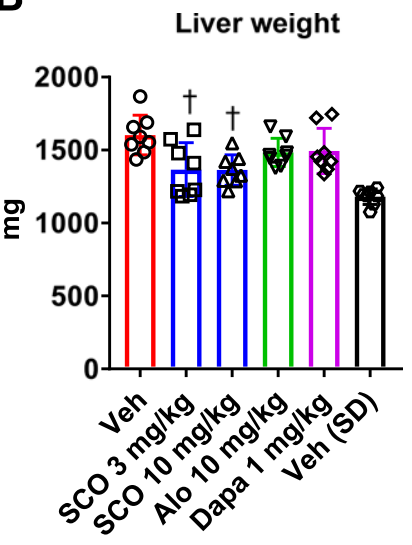

D

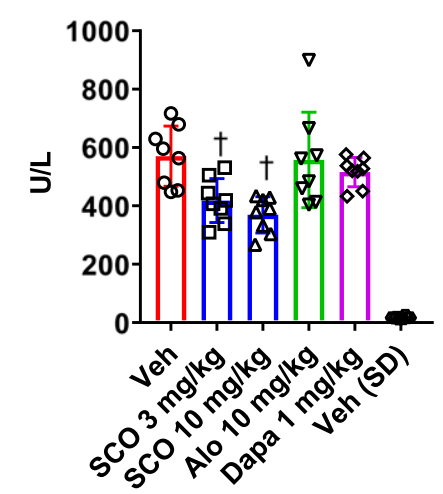

Fig. 3. Effects of repeated doses of SCO-267 on liver parameters in CDAHFD-fed mice. (A) Liver triglyceride content, (B) liver weight, (C) liver collagen content, and (D) plasma alanine aminotransferase levels at the end of the 4-week study. SCO-267 decreased liver triglyceride content, liver weight, liver collagen content, and plasma alanine aminotransferase levels. Values are presented as means \pm S.D. $(n=8) .{ }^{\dagger} P<0.025$ vs. vehicle by one-tailed Williams' test. Alo, alogliptin benzoate; Dapa, dapagliflozin; SCO, SCO-267; SD, standard diet; Veh, vehicle.

oxidative stress is likely to be a highly effective strategy to treat NAFLD in patients. The present study shows that SCO267 decreased liver oxidative stress levels, as evidenced by the decreased expression of mRNAs contributing to the generation of reactive oxygen species and the reduced TBARS level in the liver of CDAHFD-fed mice. Liver fat accumulation generates oxidative stress, which results in hepatic endoplasmic reticulum stress and inflammation (Spahis et al., 2017). Thus, the decrease in liver fat accumulation induced by SCO-267 treatment may have resulted in lower oxidative stress levels in the liver.

The addition of GLP-1 receptor agonism to glucagon receptor agonism improves lipid metabolism and hepatic steatosis when compared with GLP-1 receptor agonism alone in rodents (Day et al., 2009). In fact, a GLP-1 receptor agonist and a glucagon receptor/GLP-1 receptor coagonist administered via injection are being evaluated for the indication of NASH in a clinical trial (Knudsen and Lau, 2019; Patil et al., 2020). Considering the stimulation of glucagon and GLP-1 by GPR40 full agonists, orally available SCO-267 may be an attractive strategy to treat NASH. However, the contribution of SCO-267-induced glucagon and GLP-1 stimulation to the alleviation of liver disease warrants further study.
The present study compares the effects of SCO-267 on liver-related parameters with those of alogliptin or dapagliflozin, both clinically available antidiabetic drugs. In contrast to SCO-267, 4-week dosing of alogliptin or dapagliflozin failed to improve fatty liver and related liver parameters. Although mRNA levels of a subset of the molecules of interest were regulated by alogliptin or dapagliflozin, effects induced by each treatment were not as prominent as those induced by SCO-267 treatment. These results suggest that neither dipeptidyl peptidase-4 inhibition nor sodium glucose cotransporter 2 inhibition may be effective methods to improve the current NAFLD condition associated with normal glycemic levels.

Recently, RLA8, a quadruple agonist for peroxisome proliferator activated receptor- $\alpha / \gamma / \delta$ and GPR40, was shown to have therapeutic efficacy in NASH mouse models (Li et al., 2019). This suggests that a strategy to activate multiple targets will likely prove to be effective in treating NASH. Unlike GPR40 partial agonist, full agonism by SCO-267 has the ability to secrete glucagon and GLP-1 in addition to other islet and gut hormones (Ueno et al., 2019). Through these actions, SCO-267 likely activates multiple targets. Thus, full agonism of GPR40 may serve as a novel strategy to activate multiple targets for the treatment of NASH.

The primary limitation of this study is the lack of a longterm investigation of SCO-267 using the NAFLD mouse model. The present study evaluated the effects of 4 -week SCO-267 treatment in mice fed a CDAHFD for 5 weeks. Thus, the effects of SCO-267 on a more advanced disease condition remain unclear. Matsumoto et al. (2013) reported that up to 6-12 weeks of feeding with CDAHFD was necessary to induce histochemically observable fibrosis in CDAHFD-fed mice, implying that the present study evaluated the treatment efficacy of SCO-267 in an early phase of NAFLD. Hence, longer study duration would be essential to fully understand how GPR40 full agonists act on the disease spectrum of a preclinical NAFLD model. In addition, although SCO267-mediated stimulation of glucagon and GLP-1 secretion likely contribute to the treatment efficacy, the detailed mechanisms linking GPR40 full activation to the observed

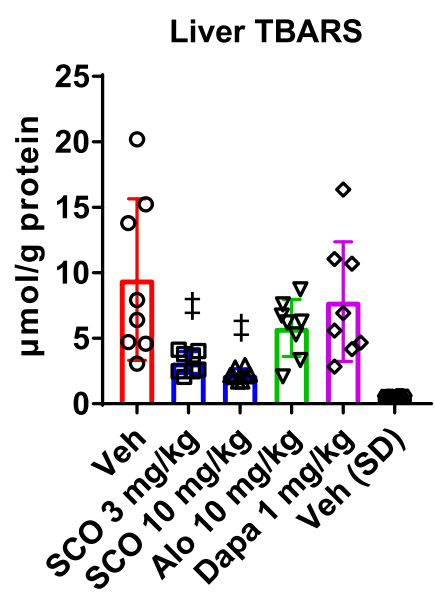

Fig. 4. Effects of repeated doses of SCO-267 on liver TBARS levels in CDAHFD-fed mice. Liver TBARS levels at the end of the 4-week study. SCO-267 decreased liver TBARS levels. Values are presented as means \pm S.D. $(n=8) .{ }^{*} P<0.025$ vs. vehicle by one-tailed Shirley-Williams test. Alo, alogliptin benzoate; Dapa, dapagliflozin; SCO, SCO-267; SD, standard diet; Veh, vehicle. 


\section{B Beta-oxidation}

\section{A Mitochondrial function}

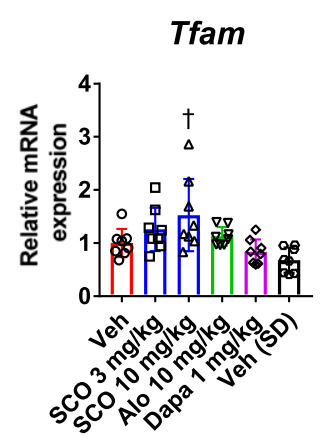

\section{Lipogenesis}
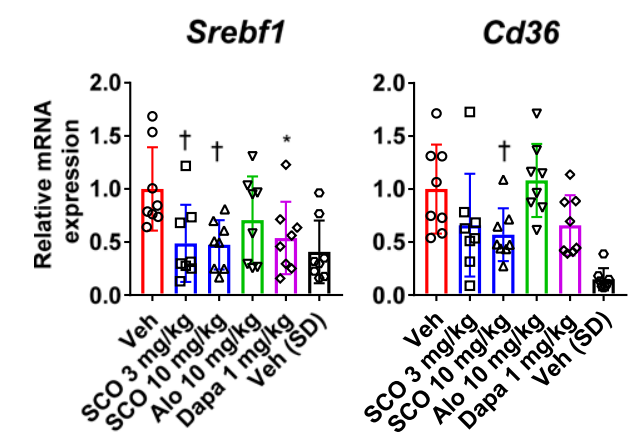

\section{E Reactive oxygen species generation}

\section{Ncf1}

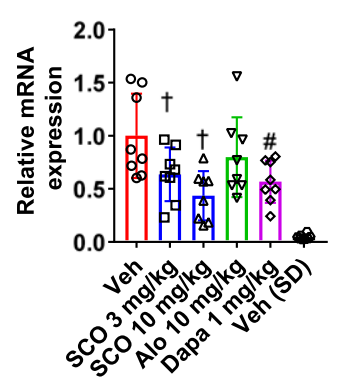

Cybb
Ppara
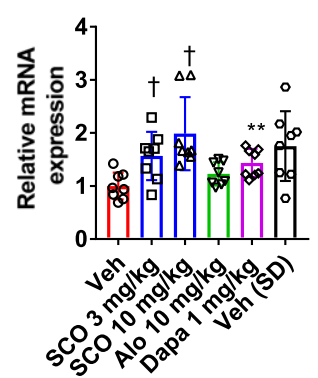

D Inflammation

Tnf

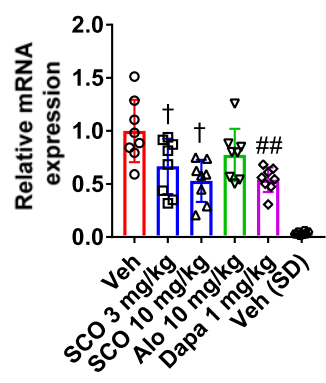

116
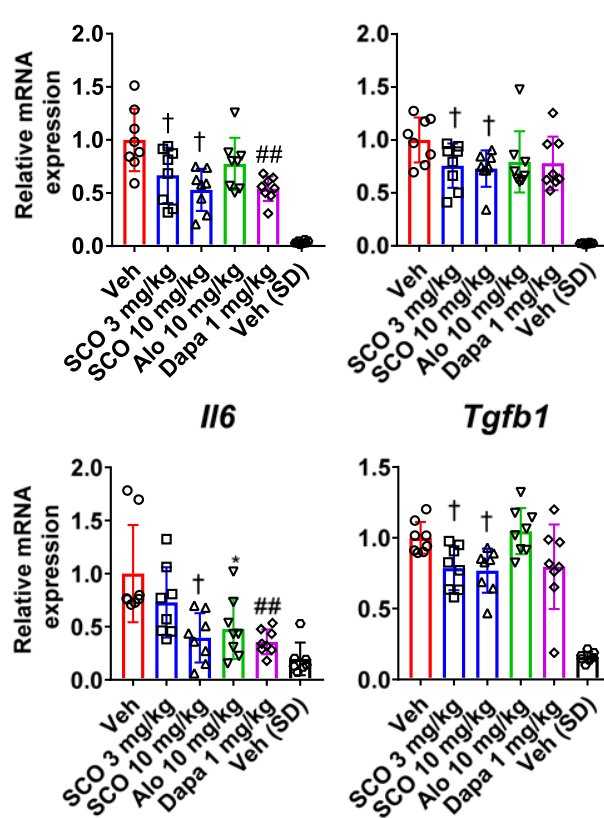

F Fibrosis

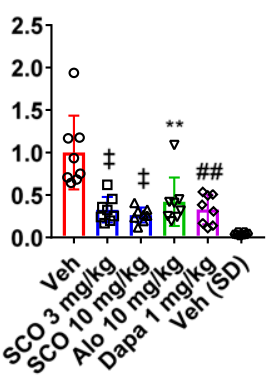

Col1a1

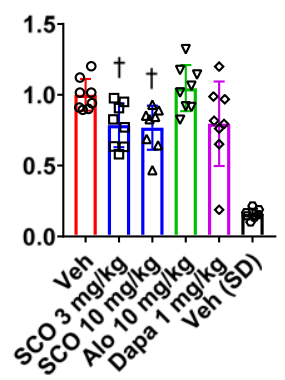

Acta2

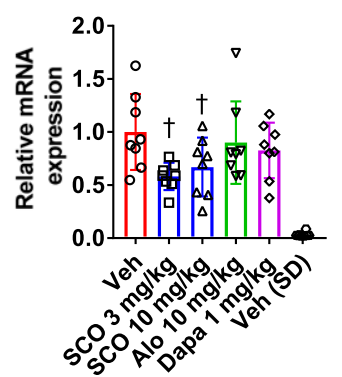

Acadl

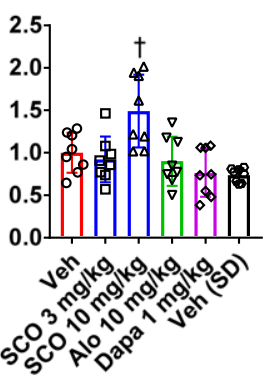

Cc/2

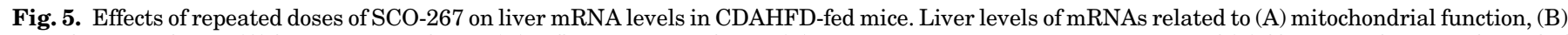

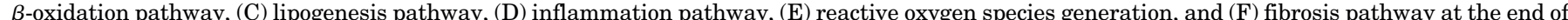

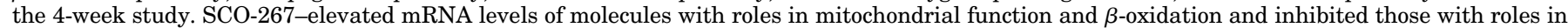

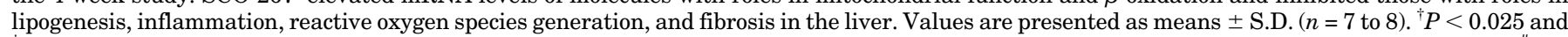

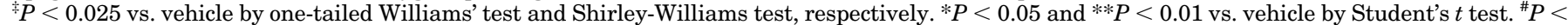

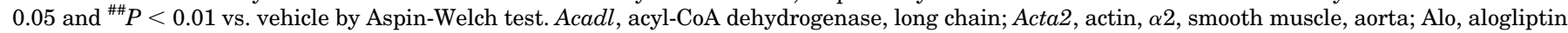

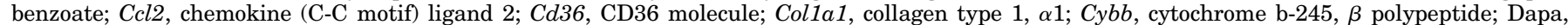

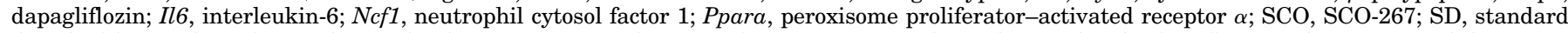

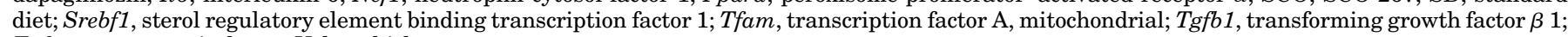
$T n f$, tumor necrosis factor; Veh, vehicle. 
efficacy remain unclear, and this should be investigated in the future.

In conclusion, the GPR40 full agonist SCO-267 stimulated glucagon and GLP-1 secretion in a mouse model of NAFLD. This is the first study to demonstrate that repeated administration of SCO-267 results in improved liver conditions in a nondiabetic mouse model of early-stage NAFLD. Notably, these effects were induced without any effects on glucose levels or body weight. Taken together, our findings demonstrate that SCO-267-mediated full activation of GPR40 was effective in alleviating NAFLD in mice and may induce similar treatment effects in patients.

\section{Acknowledgments}

We thank Akihiro Kobayashi for measuring pharmacokinetic parameters. We gratefully acknowledge Hinako Kooriyama for support.

\section{Authorship Contributions}

Participated in research design: Ookawara, Watanabe, Moritoh.

Conducted experiments: Ookawara, Matsuda, Moritoh.

Performed data analysis: Ookawara, Matsuda, Watanabe, Moritoh.

Wrote or contributed to the writing of the manuscript: Moritoh.

\section{References}

Arab JP, Arrese M, and Trauner M (2018) Recent insights into the pathogenesis of nonalcoholic fatty liver disease. Annu Rev Pathol 13:321-350.

Armstrong MJ, Gaunt P, Aithal GP, Barton D, Hull D, Parker R, Hazlehurst JM, Guo K, Abouda G, Aldersley MA, et al.; LEAN trial team (2016) Liraglutide safety and efficacy in patients with non-alcoholic steatohepatitis (LEAN): a multicentre, double-blind, randomised, placebo-controlled phase 2 study. Lancet 387:679-690.

Cohen JC, Horton JD, and Hobbs HH (2011) Human fatty liver disease: old questions and new insights. Science 332:1519-1523.

Day JW, Ottaway N, Patterson JT, Gelfanov V, Smiley D, Gidda J, Findeisen H, Bruemmer D, Drucker DJ, Chaudhary N, et al. (2009) A new glucagon and GLP-1 co-agonist eliminates obesity in rodents. Nat Chem Biol 5:749-757.

Filippatos TD, Liberopoulos EN, and Elisaf MS (2015) Dapagliflozin in patients with type 2 diabetes mellitus. Ther Adv Endocrinol Metab 6:29-41.

Ghislain J and Poitout V (2017) The role and future of FFA1 as a therapeutic target. Handb Exp Pharmacol 236:159-180.

Kaku K, Enya K, Nakaya R, Ohira T, and Matsuno R (2016) Long-term safety and efficacy of fasiglifam (TAK-875), a G-protein-coupled receptor 40 agonist, as monotherapy and combination therapy in Japanese patients with type 2 diabetes: a $52-$ week open-label phase III study. Diabetes Obes Metab 18:925-929.

Keating GM (2015) Alogliptin: a review of its use in patients with type 2 diabetes mellitus. Drugs 75:777-796.

Knudsen LB and Lau J (2019) The discovery and development of liraglutide and semaglutide. Front Endocrinol (Lausanne) 10:155.

Kothari S, Dhami-Shah H, and Shah SR (2019) Antidiabetic drugs and statins in nonalcoholic fatty liver disease. J Clin Exp Hepatol 9:723-730.
Li MH, Chen W, Wang LL, Sun JL, Zhou L, Shi YC, Wang CH, Zhong BH, Shi WG, and Guo ZW (2019) RLA8-A new and highly effective quadruple PPAR- $\alpha / \gamma / \delta$ and GPR40 agonist to reverse nonalcoholic steatohepatitis and fibrosis. J Pharmacol Exp Ther 369:67-77.

Mancini AD and Poitout V (2013) The fatty acid receptor FFA1/GPR40 a decade later: how much do we know? Trends Endocrinol Metab 24:398-407.

Matsumoto M, Hada N, Sakamaki Y, Uno A, Shiga T, Tanaka C, Ito T, Katsume A, and Sudoh M (2013) An improved mouse model that rapidly develops fibrosis in non-alcoholic steatohepatitis. Int J Exp Pathol 94:93-103.

McMurray JJV, Solomon SD, Inzucchi SE, Køber L, Kosiborod MN, Martinez FA, Ponikowski P, Sabatine MS, Anand IS, Bělohlávek J, et al.; DAPA-HF Trial Committees and Investigators (2019) Dapagliflozin in patients with heart failure and reduced ejection fraction. N Engl J Med 381:1995-2008.

Musso G, Cassader M, and Gambino R (2016) Non-alcoholic steatohepatitis: emerging molecular targets and therapeutic strategies. Nat Rev Drug Discov 15:249-274.

Pais R, Gribble FM, and Reimann F (2016) Stimulation of incretin secreting cells. Ther Adv Endocrinol Metab 7:24-42.

Patel V, Joharapurkar A, Kshirsagar S, Patel M, Sutariya B, Patel H, Pandey D, Patel D, Ranvir R, Kadam S, et al. (2018) Coagonist of glucagon-like peptide-1 and glucagon receptors ameliorates nonalcoholic fatty liver disease. Can $J$ Physiol Pharmacol 96:587-596.

Patil M, Deshmukh NJ, Patel M, and Sangle GV (2020) Glucagon-based therapy: past, present and future. Peptides 127:170296.

Sanyal AJ, Chalasani N, Kowdley KV, McCullough A, Diehl AM, Bass NM, Neuschwander-Tetri BA, Lavine JE, Tonascia J, Unalp A, et al.; NASH CRN (2010) Pioglitazone, vitamin E, or placebo for nonalcoholic steatohepatitis. $N$ Engl J Med 362:1675-1685.

Seghieri M, Christensen AS, Andersen A, Solini A, Knop FK, and Vilsbøll T (2018) Future perspectives on GLP-1 receptor agonists and GLP-1/glucagon receptor coagonists in the treatment of NAFLD. Front Endocrinol (Lausanne) 9:649.

Sinclair EM, Yusta B, Streutker C, Baggio LL, Koehler J, Charron MJ, and Drucker DJ (2008) Glucagon receptor signaling is essential for control of murine hepatocyte survival. Gastroenterology 135:2096-2106.

Spahis S, Delvin E, Borys JM, and Levy E (2017) Oxidative stress as a critical factor in nonalcoholic fatty liver disease pathogenesis. Antioxid Redox Signal 26:519-541.

Svoboda M, Tastenoy M, Vertongen P, and Robberecht P (1994) Relative quantitative analysis of glucagon receptor mRNA in rat tissues. Mol Cell Endocrinol 105: 131-137.

Tahara A, Takasu T, Yokono M, Imamura M, and Kurosaki E (2016) Characterization and comparison of sodium-glucose cotransporter 2 inhibitors in pharmacokinetics, pharmacodynamics, and pharmacologic effects. J Pharmacol Sci 130 $159-169$.

Uchii M, Kimoto N, Sakai M, Kitayama T, and Kunori S (2016) Glucose-independent renoprotective mechanisms of the tissue dipeptidyl peptidase-4 inhibitor, saxagliptin, in Dahl salt-sensitive hypertensive rats. Eur J Pharmacol 783:56-63.

Ueno H, Ito R, Abe SI, Ookawara M, Miyashita H, Ogino H, Miyamoto Y, Yoshihara T, Kobayashi A, Tsujihata Y, et al. (2019) SCO-267, a GPR40 full agonist, improves glycemic and body weight control in rat models of diabetes and obesity. J Pharmacol Exp Ther 370:172-181.

Wattacheril J, Issa D, and Sanyal A (2018) Nonalcoholic Steatohepatitis (NASH) and hepatic fibrosis: emerging therapies. Annu Rev Pharmacol Toxicol 58:649-662.

Yagi K (1998) Simple assay for the level of total lipid peroxides in serum or plasma. Methods Mol Biol 108:101-106.

Zhang X, Wang Z, Huang Y, and Wang J (2011) Effects of chronic administration of alogliptin on the development of diabetes and $\beta$-cell function in high fat diet/ streptozotocin diabetic mice. Diabetes Obes Metab 13:337-347.

Address correspondence to: Yusuke Moritoh, Research and Development Division, SCOHIA PHARMA, Inc., 26-1, Muraoka-Higashi 2-chome, Fujisawa, Kanagawa 251-8555, Japan. E-mail: yusuke.moritoh@scohia.com 